\title{
Regulatory Flexibilities and Guidances for Addressing the Challenges of COVID-19 in the EU: What Can We Learn from Company Experiences?
}

\author{
Kevin Klein ${ }^{1}$ (D) Pieter Stolk ${ }^{1} \cdot$ Pär Tellner $^{2} \cdot$ Virginia Acha $^{3} \cdot$ Sarah Montagne $^{4} \cdot$ Isabelle Stöckert $^{5}$
}

Received: 6 October 2021 / Accepted: 24 January 2022 / Published online: 7 February 2022

(c) The Drug Information Association, Inc 2022

\begin{abstract}
The COVID-19 pandemic required urgency in the development and delivery of effective vaccines and therapeutics; meanwhile, ongoing clinical research, regulation and supply for other much-needed therapeutics and vaccines needed to be sustained. In Europe, the European Commission, the European Medicines Agency (EMA) and the national regulatory agencies (NRAs) responded by issuing guidance outlining regulatory flexibilities mainly directed at COVID-19 vaccines and, belatedly, therapeutics. Using a survey methodology, this study gathered the views of the R\&D based pharmaceutical industry in May-June 2021 on the value of these flexibilities for continued use in the post-pandemic era as well as for future use in health emergency situations. Findings indicate that many flexibilities were foreseen to have value beyond the pandemic, particularly where EU and Member States aligned closely to provide a singular, streamlined regulatory environment. Digitalization was a notable driver of these flexibilities, but innovations in regulatory process (e.g. rolling reviews, flexible Scientific Advice) improved the process and outcomes measurably. Finally, the rapid reaction of the EU regulatory system and extensive efforts by all involved in providing innovative therapeutics and vaccines to patients in need provides learnings for the upcoming overhaul of the pharmaceutical acquis.
\end{abstract}

Keywords Regulatory flexibilties · COVID-19 · Clinical research · Quality and supply · Pharmacovigilance

\section{Introduction}

The coronavirus disease 2019 (COVID-19) pandemic has impacted all aspects of development, manufacturing, authorisation and pharmacovigilance of medicines and vaccines in Europe. As the pandemic escalated, the European regulatory authorities recognised the need to introduce regulatory flexibilities $^{1}$ to assure the continuity of activities so that scientific progress is not impeded, and patients continue to have access to much-needed vaccines and treatments. Furthermore,

Kevin Klein

kevin@exon-consultancy.nl

Exon Consultancy, Amsterdam, The Netherlands

2 European Federation of Pharmaceutical Industries and Associations (EFPIA), Ixelles, Belgium

3 MSD, London, UK

4 Bayer PLC, Reading, UK

5 Bayer AG, Wuppertal, Germany authorities from the European Union (EU) introduced regulatory flexibilities to support the development and evaluation of COVID-19 therapeutics and vaccines [1]. To this end, in May of 2021 the European Commission (EC) also acknowledged the need for a concerted effort by introducing the "EU strategy on COVID-19 therapeutics" [2].

Since the announcement of the pandemic, a natural stocktaking is underway in many fields to understand what we have learned from this health emergency [3-6]. As such, the COVID-19 pandemic has acted as a natural experiment providing a learning opportunity for regulation in the postpandemic era and for future public health emergencies. In this paper we conducted an assessment of the experiences

\footnotetext{
1 The European Union authorities used the term "regulatory flexibilities"; however, the WHO has described these as "regulatory agilities". Both terms refer to the same regulatory arrangements.
} 
of $R \& D$ based pharmaceutical companies with the regulatory flexibilities introduced by EU authorities. ${ }^{2}$ The aim of this study was to gather views, experiences and learnings of pharmaceutical companies on how these regulatory flexibilities could be considered for future use in either the business as usual setting or pandemic preparedness of the EU regulatory system.

Therefore, this study has the following main objectives:

(1) Regulatory flexibilities to assure the continuity of supply of medicines To assess the value and impact of the regulatory flexibilities in support of supplying medicines, and to what extent these flexibilities could be considered for continued use in the post-pandemic era ("business as usual").

(2) Regulatory flexibilities and other measures to support the development of COVID-19 therapeutics To assess the value of the regulatory flexibilities and measures for accelerating/supporting the development of COVID-19 therapeutics and to what extent these flexibilities could be considered for future use in health emergency situations ("pandemic preparedness").

A separate study has been conducted by Vaccines Europe to collect input from COVID-19 vaccine developers, and therefore questions related to regulatory flexibilities for COVID-19 vaccine development were not in scope of this analysis and will be addressed in different fora.

\section{Methods}

We designed a digital survey to address the objectives of this study which contained two parts:

- Survey part A to meet objective 1: contains questions to gather the views, experiences and learnings of R\&D based pharmaceutical companies about the implemented regulatory flexibilities aimed to address various challenges arising from the COVID-19 pandemic in order to ensure continuity of the development and supply of medicines. This section also covers regulatory flexibilities specifically introduced for crucial medicines for treatment of COVID-19 patients, to gather views on their potential value for continued use in a broader scope.

- Survey part B to meet objective 2: contains questions about the value of regulatory flexibilities for supporting/ accelerating the development of COVID-19 therapeutics,

\footnotetext{
2 The survey is a follow-up to a previous survey conducted by EFPIA in September 2020 to collect data on the initial experiences of EFPIA member companies with regulatory flexibilities during the COVID19 pandemic.
}

only for EFPIA member companies who are/were developing or have received authorisation for a COVID-19 therapeutic.

The questions of the survey are based on the regulatory flexibilities detailed in relevant guidance documents from the EC, the European Medicines Agency (EMA), Heads of Medicines Agencies (HMA), the Co-ordination Group for Mutual Recognition and Decentralised Procedures-human (CMDh) and also EU regulations. The survey instrument is spreadsheet-based and divided into two workbooks for parts A and B. Companies could only have one response to parts A and B; however, they were invited to collate input from subject matter experts across the company. Although this multidisciplinary input was recorded in company responses, informal EFPIA exchanges suggested that this was common practice for most.

Regulatory guidance and flexibilities included in both survey's part A and B:

- Notice to stakeholders: Questions and answers on regulatory expectations for medicinal products for human use during the COVID-19 pandemic (Rev. 3) [7]

- CMDh Practical guidance (CMDh/418/2020, Rev. 1) [8]

- Guidance on the management of clinical trials during the COVID-19 (coronavirus) pandemic (Version 4 from July 2021) [9]

- Regulation (EU) 2020/1043 (July 2020) [10]

Additional regulatory flexibilities and EMA initiatives for accelerating/supporting the development of COVID-19 therapeutics that were included in survey part B:

- Mandate, objectives and rules of procedure of the COVID19 EMA pandemic Task Force (COVID-ETF) [11]

- EMA initiatives for acceleration of development support and evaluation procedures for COVID-19 treatments and vaccines [12]

- Labelling flexibilities for COVID-19 therapeutics [13]

- FDA/EMA common commentary on submitting an initial Pediatric Study Plan (iPSP) and Paediatric Investigation Plan (PIP) for the prevention and treatment of COVID-19 [14]

In survey part $\mathrm{A}$, we asked participants to score regulatory flexibilities with regard to their value for continued use in a post-pandemic era with a four-point Likert scale: 0 -no value, 1 -limited value, 2 - some value and 3-significant value. Respondents were also able to abstain from answering, e.g. in case of no/limited experience with a particular flexibility. By selecting an even score Likert scale, the survey design omits the midpoint to encourage a clear response, whilst allowing a neutral or unfamiliar response ('Don't know') as recommended [15]. 
In survey part $\mathrm{B}$, we asked participants to score regulatory flexibilities with regard to their value for supporting/ accelerating the development of COVID-19 therapeutics. The same four-point Likert scale was used: 0-no value, 1-limited value, 2-some value and 3-significant value, with the possibility to abstain from answering.

Both surveys comprised of a general questions section followed by questions on the regulatory flexibilities along four themes: (i) regulatory, (ii) quality and supply, (iii) clinical research and (iv) pharmacovigilance. The questions consisted of a mix of multiple choice questions and free text fields to provide details. An additional set of multiple choice questions and free text fields was included in survey part B in which respondents could indicate their positive and negative experience with regulatory flexibilities. This allowed for a structured analysis of the free text fields. The survey was circulated to $57 \mathrm{R} \& \mathrm{D}$ based pharmaceutical companies, including EFPIA member companies, small and mediumsized enterprises (SMEs) and EFPIA partners in research. Of these 57 companies, 18 companies were involved in the development of COVID-19 therapeutics and therefore also invited to respond to survey part B (according to the EMA database as of 1 July 2021) [16]. ${ }^{3}$ The survey was circulated via email to senior company representatives. Respondents were given a response time of 6 weeks, from April 29th to June 10th 2021. A reminder was sent after five weeks. Data was consolidated and analysed in Microsoft Excel®. Only authors KK and PS, who were not employed or contracted by an EFPIA member-company, had access to the identity of the respondents, this was also communicated to the survey participants. The full survey materials for part A and B can be found in supplemental section.

\section{Results}

\section{General Results}

A total of 17 out of 57 (30\%) companies responded to survey part A. A total of eight out of 18 (44\%) companies that were involved in developing COVID-19 therapeutics responded to survey part B. Of these eight Part B responding companies, two companies indicated to have a COVID-19 therapeutic under rolling review in the EU, seven indicated to have COVID-19 treatments under development, and five respondents indicated to have at least one COVID-19 therapeutic for which the development has stopped. None of the respondents

\footnotetext{
${ }^{3}$ I.e. companies that had a COVID-19 therapeutic approved, under marketing authorisation (MA) evaluation, under rolling review (RR), or sought EMA advice.
}

had yet received approval for a COVID-19 therapeutic in the EU at the time of the survey response.

\section{The Results of Survey Part A}

\section{Regulatory Section}

The flexibilities in this "Regulatory" section of survey Part A received 'mixed' responses, with some respondents indicating significant value, whereas other respondents indicated limited or even no value for continued use (Table 1).

The regulatory flexibilities on variations (A1.1) and on language and labelling (A1.4) scored highest in this section with $38 \%$ and $31 \%$ of the responses respectively indicating significant value and the majority of the remaining responses indicating some value. It was noted that the procedures for speeding up variations, when justified, provide better opportunities to secure continuous supply to all markets. Language and labelling flexibilities were generally welcomed by companies, as these flexibilities could be valuable to ensure continued patients access to products with severe supply constraints. It was however pointed out that a lack of harmonisation between EU Member States limits the value of both flexibilities, and therefore should be addressed to maximize the potential of these flexibilities for continued use in the post-pandemic era.

The flexibilities related to zero-day mutual recognition (MR)/repeat use (RU) procedures and assessment timelines (A1.2) were considered of less value for continued use in the post-pandemic era. It was noted that more specific guidance is needed to make better use of zero-day MR/RU procedures. The flexibilities on renewals (A1.3) scored low as well when it comes to their value for continued use in the post-pandemic era, as there was no or limited regulatory value in applying these flexibilities in anything other than in exceptional circumstances such as a pandemic.

\section{Quality and Supply Section}

The flexibilities from the quality and supply section received the most responses of all four sections, with 13 responses on average and some of the highest valued flexibilities recorded by respondents to Part A.

The flexibilities and guidances regarding Exceptional Change Management Process (ECMP) (A2.1), Good Manufacturing Practice (GMP) certification (A2.3) and Qualified Person (QP)/Responsible Person (RP) remote working (A2.4) scored highest in this section. They also scored highly compared to the other sections in Part A, with $44 \%$, $62 \%$ and $64 \%$ of the respondents respectively indicating a significant value in the continued use of these flexibilities in the post-pandemic era. Furthermore, there was general consensus as none of the respondents rated either of these 
Table 1 Overview of Responses to Flexibilities and Guidances from Survey Part A Related to Regulatory (A1), Quality and Supply (A2), Clinical Research (A3) and Pharmacovigilance (A4) Activities with
Regard to Their Value for Continued Use in the Post-pandemic Era $($ Total Number of Respondents $=17)$

\begin{tabular}{|c|c|c|c|c|c|c|}
\hline & $\begin{array}{l}3 \text {-Significant } \\
\text { Value }\left(N\left[\%^{\mathrm{a}}\right]\right)\end{array}$ & $\begin{array}{c}2 \text {-Some } \\
\text { Value }\left(N\left[\% \%^{\mathrm{a}}\right]\right)\end{array}$ & $\begin{array}{l}1 \text {-Limited } \\
\text { Value }\left(N\left[\%^{\mathrm{a}}\right]\right)\end{array}$ & $\begin{array}{c}0-\text { No Value } \\
\left(N\left[\%{ }^{\mathrm{a}}\right]\right)\end{array}$ & $\begin{array}{l}\text { Responders } \\
\left(N\left[\%^{\mathrm{b}}\right]\right)\end{array}$ & Average Score \\
\hline A1.1-Variations & $5(38)$ & $6(46)$ & $2(15)$ & $0(0)$ & $13(76)$ & 2.2 \\
\hline $\begin{array}{l}\text { A1.2-Zero-day MR/RU procedures and } \\
\text { assessment timelines }\end{array}$ & $2(22)$ & $3(33)$ & $3(33)$ & $1(9)$ & $9(53)$ & 1.7 \\
\hline A1.3-Renewals & $1(9)$ & $3(27)$ & $5(45)$ & $2(18)$ & $11(65)$ & 1.3 \\
\hline A1.4-Language and labelling & $4(31)$ & $7(54)$ & $2(15)$ & $0(0)$ & $13(76)$ & 2.2 \\
\hline A2.1-ECMP & $4(44)$ & $5(56)$ & $0(0)$ & $0(0)$ & $9(53)$ & 2.4 \\
\hline A2.2-Quality variations & $2(20)$ & $4(40)$ & $3(30)$ & $1(10)$ & $10(59)$ & 1.7 \\
\hline A2.3-GMP certifications & $8(62)$ & $5(38)$ & $0(0)$ & $0(0)$ & $13(76)$ & 2.6 \\
\hline A2.4-QP/RP remote working & $7(64)$ & $4(36)$ & $0(0)$ & $0(0)$ & $11(65)$ & 2.6 \\
\hline A2.5-Importation testing & $4(40)$ & $1(10)$ & $5(50)$ & $0(0)$ & $10(59)$ & 1.9 \\
\hline A3.1-Amendments & $3(23)$ & $7(54)$ & $3(23)$ & $0(0)$ & $13(76)$ & 2.0 \\
\hline A3.2-Informed consent & $3(21)$ & $7(50)$ & $4(29)$ & $0(0)$ & $14(82)$ & 1.9 \\
\hline A3.3-Direct-to-patient delivery & $9(60)$ & $3(20)$ & $3(20)$ & $0(0)$ & $15(88)$ & 2.4 \\
\hline A3.4-Alternative trial/lab sites & $2(15)$ & $9(69)$ & $2(15)$ & $0(0)$ & $13(76)$ & 2.0 \\
\hline A3.5-Remote source data verification & $7(47)$ & $6(40)$ & $2(13)$ & $0(0)$ & $15(88)$ & 2.3 \\
\hline A3.6-GMO derogation & $6(67)$ & $1(11)$ & $2(22)$ & $0(0)$ & $9(53)$ & 2.4 \\
\hline A4.1-Safety reporting & $2(20)$ & $5(50)$ & $3(30)$ & $0(0)$ & $10(59)$ & 1.9 \\
\hline A 4.2 - System audits & $0(0)$ & $8(80)$ & $2(20)$ & $0(0)$ & $10(59)$ & 1.8 \\
\hline A4.3-Remote inspections & $4(36)$ & $4(36)$ & $3(27)$ & $0(0)$ & $11(65)$ & 2.1 \\
\hline A4.4-CAPA management & $2(18)$ & $6(55)$ & $3(27)$ & $0(0)$ & $11(65)$ & 1.9 \\
\hline
\end{tabular}

$N$ number of responses

$\mathrm{MR} / \mathrm{RU}=$ Mutual Recognition/Repeat Use; ECMP=Exceptional Change Management Process; GMP=Good Manufacturing Practice; QP/ $\mathrm{RP}=$ Qualified Person/Responsible Person; GMO = Genetically Modified Organisms; CAPA = Corrective and Preventative Actions

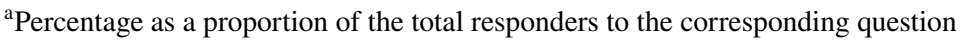

${ }^{b}$ Percentage as a proportion of the total number of respondents to survey part A $(=17)$

flexibilities with 'limited' or 'no value'. The ECMP flexibility was considered particularly valuable for continued use in the post-pandemic era to address supply constraints and avoiding drug shortages, if the scope of the ECMP is extended to other products, for example to include essential medicines for life threatening conditions. It was however pointed out that ECMP could benefit from process simplification and a broader scope that covers all aspects of the manufacturing process, control strategy and supply chain. The flexibilities on remote assessments were considered to be of high value for the post-pandemic era, both for extending validity of GMP certificates as well as to authorise new sites, which can also help to ensure continuity of manufacture and supply. But it was also commented that more harmonisation between EU Member States is needed to make best use of this flexibility. The use of digital technologies that enable QP/RP remote working were also welcomed by the respondents, who generally expressed keen interest in the continued use of digital technologies enabling remote working.

The flexibilities regarding quality variations (A2.2) and importation testing (A2.5) scored lower compared to the other flexibilities from this section, with $30 \%$ and $50 \%$ of the responses respectively indicating only limited value for continued use of these flexibilities. Although respondents appreciated the ability to engage with the authorities for waivers of quality requirements, it was pointed out that the procedures for quality variations were not particularly practical. The flexibilities on importation testing, which allows in justified cases to postpone requirements for importation testing, were also not considered very valuable. Although the option to waive importation testing was welcomed, respondents indicated that the process of importation testing needs more fundamental reconsiderations to address redundant importation testing in the future. Although not directly addressed in their comments, this response surely reflects recent publications that outline the opportunities for change $[17,18]$. 


\section{Clinical Research Section}

The flexibilities from the clinical research section received mixed responses. Responses to all six flexibilities ranged from limited value to significant value.

The flexibilities related to direct-to-patient delivery (A3.3), remote Source Data Verification (rSDV) (A3.5) and Genetically Modified Organisms (GMO) derogation (A3.6) scored highest in this section. The flexibilities allowing direct-to-patient delivery were considered valuable for continued use in the post-pandemic era to further improve and enrich the clinical research environment in Europe. This flexibility provided benefits to the patients by limiting unnecessary site visits, which even outside of a pandemic setting, can be burdensome for participants. Moreover, direct-to-patient delivery is also particularly valuable for decentralized clinical trials, ${ }^{4}$ which is a new approach to clinical research which aims to improve efficiencies for the benefit of all stakeholders. However, it was also commented that this flexibility is of less value for more complex administrations (e.g. IV products). It was indicated that the flexibility on rSDV can have an important value in the postpandemic era, especially in the case of decentralised clinical trials. However, it was also pointed out that more guidance is needed on cyber security and protection of individual data, and the scope of the rSDV flexibility should be broadened to all clinical trials. Despite only nine responses, two-third indicated a significant value for applying GMO derogations in the post-pandemic era if the scope is extended beyond the scope of products intended to treat or prevent COVID19. It was pointed out by some respondents that extending GMO derogation to all Advanced Therapy Medicinal Products (ATMPs) in clinical development in the EU would be a major benefit for ATMP developers, reducing delays associated with the submission of environmental risk assessments. One limitation of the GMO derogation that was pointed out, referred to the lack of harmonisation between MS regarding the interpretation and application of GMO legislation at the national level, which needs to be resolved to make best use of GMO derogations in the future.

The flexibilities on amendments (A3.1), informed consent (A3.2) and alternative trial/lab sites (A3.4) scored lower compared to the other flexibilities from this section, averaging a score of two ('some value') on the four-point scale. Although the flexibility on amendments was considered valuable by some respondents, it was mentioned the lack of harmonisation between EU Member States limits the value of this flexibility. The flexibilities for alternative ways to

\footnotetext{
${ }^{4}$ For further details on decentralized clinical trials, please refer to the Clinical Trials Transformation Initiative-CTTI (ctti-clinicaltrials. org).
}

obtain informed consent were also welcomed, however it was indicated by some respondents that the guidance needs be revised to formally accept electronic consent (e.g. using a validated system) and improve remote re-consent to maximize the value of this flexibility. The lack of harmonisation between EU Member States in applying this flexibility also made it difficult to use. The flexibility of alternative trial/lab sites was considered to have some value in the future, however more efforts are needed to minimise the administrative burden regarding registration of trial sites and labs.

\section{Pharmacovigilance Section}

The flexibilities from the pharmacovigilance section scored near an average of 2 ('some value'). The guidance and flexibilities on safety reporting (A4.1) helped to prioritise the processing of serious adverse events (AEs) and adverse events of special interest (AESIs) related to COVID-19 therapeutics and deprioritise non-serious AEs. However, it was indicated that further guidance on flexibilities regarding timelines (e.g. 15-day for serious Individual case safety reports), in case reporting obligations could not be met, would be helpful. The guidance and flexibilities on system audits (A4.2) provided a reasonable flexibility that allowed for a risk-based approach and 'for cause' prioritisation. In general, companies responded positively to EMA accepting remote $\mathrm{PV}$ audits and the recommendations regarding remote auditing was considered valuable. Participating companies welcomed the ability to conduct remote inspections (A4.3), as this enabled inspections to be rolled out faster and reduced the travel time. The flexibilities on risk-based prioritization for managing Corrective and Preventative Actions (CAPAs) (A4.4) helped to mitigate most significant impacts of deviations, although some respondents indicated that in some cases this was already standard practice.

\section{Other Comments and Considerations Provided in Survey}

Part A The respondents provided some additional comments on guidances and flexibilities. Some respondents indicated that more progress is needed towards e-labelling and other ways for electronic product information, which, in combination with the language \& labelling flexibilities, could help to support the continued delivery of updated information to patients in such circumstances. It was generally commented that many guidances and flexibilities could be applied in the post-pandemic era to address drug shortages and supply constraints. The use of digital technologies in other regulatory areas was also considered helpful, for example the continued use of the flexibility offered by EMA for conducting virtual meetings (e.g. scientific advice, oral explanation), or the introduction of a standardized e-signature process and certification (e.g. electronic Certificates of Pharmaceutical Products [eCPPs]) for pharmaceutical companies and rele- 
Table 2 Overview of Responses to Flexibilities and Guidances from Survey Part B Related to Regulatory (B1), Quality and Supply (B2), Clinical Research (B3) and Pharmacovigilance (B4) Activities with
Regard to Their Value for Accelerating/Supporting the Development of COVID-19 Therapeutics (Total Number of Respondents $=8$ )

\begin{tabular}{|c|c|c|c|c|c|c|}
\hline & $\begin{array}{l}3 \text {-Significant } \\
\text { Value }\left(N\left[\%^{\mathrm{a}}\right]\right)\end{array}$ & $\begin{array}{l}2-\text { Some } \\
\text { Value }(N \\
{\left[\%^{\mathrm{a}]}\right)}\end{array}$ & $\begin{array}{l}1 \text { - Limited } \\
\text { Value }\left(N\left[\%^{\mathrm{a}}\right]\right)\end{array}$ & $\begin{array}{l}0-\mathrm{No} \\
\text { Value }(N \\
\left.\left[\%^{\mathrm{a}}\right]\right)\end{array}$ & $\begin{array}{l}\text { Responders } \\
\left(N\left[\%^{\mathrm{b}}\right]\right)\end{array}$ & Average Score \\
\hline B1.1—EMA pandemic Task Force & $1(20)$ & $2(40)$ & $2(40)$ & $0(0)$ & $5(63)$ & 1.8 \\
\hline B1.2-Rapid scientific advice & $4(57)$ & $2(29)$ & $1(14)$ & $0(0)$ & $7(88)$ & 2.4 \\
\hline B1.3—Rolling review & $4(80)$ & $1(20)$ & $0(0)$ & $0(0)$ & $5(63)$ & 2.8 \\
\hline $\begin{array}{l}\text { B1.4-Rapid agreement of PIPs/rapid compliance } \\
\text { check }\end{array}$ & $4(80)$ & $1(20)$ & $0(0)$ & $0(0)$ & $5(63)$ & 2.8 \\
\hline B1.5-Labelling and packaging & $3(50)$ & $3(50)$ & $0(0)$ & $0(0)$ & $6(76)$ & 2.5 \\
\hline $\begin{array}{l}\text { B1.6-Extension of indication and marketing authori- } \\
\text { sation }\end{array}$ & $1(20)$ & $2(40)$ & $2(40)$ & $0(0)$ & $5(63)$ & 1.8 \\
\hline B2.1-ECMP & $1(33)$ & $2(67)$ & $0(0)$ & $0(0)$ & $3(37)$ & 2.3 \\
\hline B2.2-Quality variations & $2(50)$ & $2(50)$ & $0(0)$ & $0(0)$ & $4(50)$ & 2.5 \\
\hline B2.3-GMP certifications & $1(25)$ & $3(75)$ & $0(0)$ & $0(0)$ & $4(50)$ & 2.3 \\
\hline $\mathrm{B} 2.4-\mathrm{QP} / \mathrm{RP}$ remote working & $2(67)$ & $1(33)$ & $0(0)$ & $0(0)$ & $3(37)$ & 2.7 \\
\hline B2.5-Importation testing & $1(33)$ & $2(67)$ & $0(0)$ & $0(0)$ & $3(37)$ & 2.3 \\
\hline B3.1-Protocol amendments & $0(0)$ & $4(80)$ & $1(20)$ & $0(0)$ & $5(63)$ & 1.8 \\
\hline B3.2-Informed consent & $2(50)$ & $1(25)$ & $1(25)$ & $0(0)$ & $4(50)$ & 2.3 \\
\hline B3.3-Alternative trial/lab sites & $1(33)$ & $1(33)$ & $1(33)$ & $0(0)$ & $3(37)$ & 2.0 \\
\hline B3.4-Remote source data verification & $0(0)$ & $4(100)$ & $0(0)$ & $0(0)$ & $4(50)$ & 2.0 \\
\hline $\begin{array}{l}\text { B3.5-Initiation of new trials aiming to test new } \\
\text { treatments for COVID-19 }\end{array}$ & $0(0)$ & $4(80)$ & $1(20)$ & $0(0)$ & $5(63)$ & 1.8 \\
\hline $\begin{array}{l}\text { B3.6-Joint procedural information for submitting } \\
\text { PIP to EMA and iPSP to FDA }\end{array}$ & $0(0)$ & $3(100)$ & $0(0)$ & $0(0)$ & $3(37)$ & 2.0 \\
\hline A3.6-GMO derogation & $2(67)$ & $0(0)$ & $1(33)$ & $0(0)$ & $3(37)$ & 2.3 \\
\hline B4.1—Safety reporting & $0(0)$ & $0(0)$ & $0(0)$ & $1(100)$ & $1(12)$ & 0.0 \\
\hline B4.2-System audits & $0(0)$ & $2(67)$ & $0(0)$ & $1(33)$ & $3(37)$ & 1.3 \\
\hline B4.3-Remote inspections & $1(33)$ & $1(33)$ & $0(0)$ & $1(33)$ & $3(37)$ & 1.7 \\
\hline B4.4-CAPA management & $0(0)$ & $0(0)$ & $1(100)$ & $0(0)$ & $1(12)$ & 1.0 \\
\hline
\end{tabular}

$N$ number of responses

PIPs $=$ Paediatric Investigation Plans $; \mathrm{ECMP}=$ Exceptional Change Management Process $; \mathrm{GMP}=$ Good Manufacturing Practice; $\mathrm{QP} / \mathrm{RP}=\mathrm{Quali}-$ fied Person/Responsible Person; GMO=Genetically Modified Organisms; EMA=European Medicines Agency; iPSP=initial Pediatric Study Plan; FDA $=$ Food and Drug Administration; $\mathrm{CAPA}=$ Corrective and Preventative Actions

${ }^{a}$ Percentage as a proportion of the total responders to the corresponding question

${ }^{\mathrm{b}}$ Percentage as a proportion of the total number of respondents to survey part B $(=8)$

vant HAs where the validity can be verified instantly online. One of the major current limitations for some of the flexibilities was the lack of harmonisation between EU Member States. Companies commented that some flexibilities were only of limited value because Member States implemented these flexibilities differently.

\section{Results of Survey Part B}

\section{Regulatory Section}

The flexibilities and guidances relating to regulatory activities in developing/review and approval of COVID-19 therapeutics scored a high average (Table 2). Rolling review
(B1.3) and the rapid agreement of a Paediatric Investigation Plan (PIP) and rapid compliance check (B1.4) were considered the most valuable of all flexibilities and guidance from survey part B in supporting/accelerating the development of COVID-19 therapeutics. It was indicated by four out of five respondents that the rolling review procedure was of significant value for supporting/accelerating the development of their COVID-19 therapeutic. Respondents pointed out that the rolling review procedure provided a smooth and fast process with excellent communication between companies and the Agency. However, one respondent had different experiences and pointed out the burdensome procedures for an emergency situation, as well as unclear timelines and more documentation requirements compared to the US. The 
flexibilities on rapid agreement of a PIP and rapid compliance check were also scored 'significant value' by four out of five respondents. There were positive experiences with regards to PIP and the speed and efficiency of the procedures, although it was also noted that in some cases there is still not a complete alignment between EU and US in requirements of paediatric studies.

For the labelling and packaging flexibilities (B1.5) and rapid scientific advice (B1.2), experience was reported by six and seven companies respectively, with 50\% and 57\% (respectively) indicating significant value, although the average score is slightly lower compared to the flexibilities and guidance related to rolling review and PIPs discussed in the previous paragraph. The rapid scientific advice procedure was highly appreciated by most companies and considered very important in the context of the pandemic. However, it was indicated that there is also a need for further harmonisation between EMA and FDA to seek joint scientific advice under expedited timelines. The labelling and packaging flexibilities were considered critical in the context of global supply constraints, but it was also pointed out that the labelling and packaging guidance for 'therapeutics' was issued significantly later than for vaccines, which caused some unpredictability.

The EMA pandemic Task Force (ETF) (B1.1) and the extension of indication and marketing authorisation (MA) opportunities (B1.6) scored lowest and therefore according to the respondents seemed less valuable in supporting/accelerating the development of COVID-19 therapeutics compared to the other flexibilities and guidances. While ETF offered additional value compared to standard interactions and the consultations with ETF were considered productive and offered flexibility in terms of the documentation, two companies were critical about the timelines and/or quality of the interaction. Furthermore, in one case ETF did not remove the need to go through a formal scientific advice for issues, which was perceived as more burdensome compared to US, where companies were able to seek feedback within a matter of days to resolve outstanding issues. The flexibilities related to extension of indication and MA received mixed responses, with two out of five respondents indicating only limited value. It was commented that the basic requirements for an extension of indication was considered less effective than an emergency use procedure.

\section{Quality and Supply Section}

The flexibilities related to quality and supply activities scored high overall, with all responses indicating either significant or some value. However, it needs to be noted that experience to date of the survey (June 2021) was still limited, as only three to four responders shared experiences for each of the flexibilities in this section.
The flexibility on QP/RP remote working (B2.4) scored high overall as well, with two out of three respondents indicating a significant value in this flexibility for supporting/ accelerating the development of COVID-19 therapeutics. Flexibilities on quality variations (B2.2) also scored high, with two out of four responses indicating a significant value. It was commented that this flexibility can support the ability to offer supply of products globally at an expedited rate, for example through 'waivers' on quality controls.

The ECMP flexibilities (B2.1), GMP certifications (B2.3) and importation testing (B2.5) were all considered valuable for accelerating/supporting the development of COVID19 therapeutics, although scoring slightly lower compared to the other two flexibilities from this section. ECMP was considered valuable early engagement with regulators and agreement for GMP waivers enabled timely strategic decisions within the supply chain. However, it was remarked that more global alignment is needed since changes are not made for individual regions alone (e.g. just the EU). The flexibilities on GMP certifications were also considered valuable, in particular the automatic extension of existing GMP licences which allowed companies to secure supply of COVID-19 critical medicines to patients. One remark was made that more consideration is needed for accelerated processes for new products in obtaining a GMP certification. The flexibilities on importation testing helped to reduce overall timelines for expedited delivery of the final product ahead of completed testing. However, at the same time companies argued that a revision of the framework is needed to remove redundant importation testing.

\section{Clinical Research Section}

Most flexibilities in this section received mixed responses with responses ranging from 3 to 5 out of the total of 8 . The flexibilities related to informed consent (B3.2) and GMO derogation (B3.7) scored highest in this section, with the majority of the respondents indicating a significant value.

The flexibilities for obtaining remote informed consent were seen as valuable. GMO derogation was also considered valuable, although one respondent commented that the interpretation and application of GMO legislation at the national level varies considerably from country to country in the EU. This leads to different procedural requirements for clinical trials, different review timelines and different opinions by the national agencies which will need to be addressed, according to the respondents, in order to make best use of GMO derogations at the European level.

The other flexibilities scored on average 2 ('some value') or lower. Regarding amendments (B3.1), companies noted that the guidance was sufficient and encouraged an expedited review of amendments to COVID-19 therapeutic trials, but one company also experienced several long delays on 
protocol amendment approval, particularly from Ethic Committees. The flexibilities on alternative trial/lab sites (B3.3) had limitations as there was a lack of harmonization among EMA, FDA and other health authorities. The flexibilities on Initiation of new trials aiming to test new treatments for COVID-19 (B3.5) contained important information and encouraged an expedited review of COVID-19 therapeutic trials. However, one respondent experienced inflexibilities at the national level which delayed the resubmission of the initial CTA package.

\section{Pharmacovigilance Section}

Only few responses were received for flexibilities in the pharmacovigilance section. Overall, these flexibilities scored low, however due to the limited number of respondents (some flexibilities in this section received only one response) the results should be interpreted with care. This limited response is no doubt related to the relatively earlier stage of development of the COVID-19 therapeutics, with experience in approval, use and follow-up evaluation to come.

Only one respondent commented on the flexibilities and guidances on safety reporting (B4.1) and criticized the lack of guidance for products approved with an emergency authorisation, which resulted in Member States enforcing different requirements. It was noted that more clarity on other safety reporting requirements would be helpful. The recommendations regarding remote auditing (B4.2) were considered helpful. The acceptability of remote settings allowed for a reduction of travel time. However, it was also indicated that companies already used a range of onsite and virtual audits to support risk-based assessments of the pharmacovigilance quality system. The flexibilities on remote inspections (B4.3) was received positively providing clear instructions on inspections requests and timeline for delivery. One respondent pointed out that an opportunity was for regulators to align on a consistent framework for remote inspections, using the same platform and approach. One respondent reflected on the flexibilities and guidance on CAPA (B4.4), which were seen of limited additional value because the procedures reflected in the Q\&A document are already common practice according to that respondent [7].

\section{Other Comments and Considerations Provided in Survey} Part B The respondents to survey part B provided some additional comments on guidances and flexibilities related to the development of COVID-19 therapeutics. It was mentioned that the rolling review procedure would be a valuable addition if made available also for non-COVID related treatments. Furthermore, it was noted that the rolling review procedure could potentially be applied also for indication extensions in exceptional circumstances. Next to this, it was also commented that a separate framework for approved medicines used in unapproved indications in emergency situations could be valuable in situations like the COVID-19 pandemic. Also, it was pointed out that an EU wide emergency use authorisation (EUA) procedure would be desirable, which should enable medicine developers to initiate an EUA pathway with globally competitive timelines.

One respondent remarked that the timing of issuing guidance and Q\&As in the EU was relative slow compared to FDA, Health Canada and PMDA. Moreover, harmonisation approaches across regulatory authorities in the EU are needed to streamline the implementation and usability of guidances and flexibilities rolled out at the European level.

A remark was also made that it would be useful to have a single point of contact at the EMA if an MAH experiences difficulties and potential non-compliance issues during a pandemic.

\section{Discussion}

This survey shows the experience and assessment of pharmaceutical companies with the recently introduced regulatory flexibilities and guidances that were provided by European authorities to address challenges arising from the COVID-19 pandemic (Part A) and to accelerate and support the development of COVID-19 therapeutics (Part B). The focus of this research was to gather the views of these companies as to the value of these flexibilities for the future, both for the continued use in support of supplying medicines in the post-pandemic era ("business as usual") and future use in health emergency situations ("pandemic preparedness"). As such, the results of this study provide valuable insights that can help to improve European regulatory systems. The learnings from survey part A can contribute to improving the efficiency of European regulatory systems, while the learnings from survey part B, can contribute to strengthening the pandemic preparedness in the EU, although it has to be noted that the data set is limited in terms of numbers of respondents and experiences gathered at the time.

\section{Reflection on the Results}

The results survey part A and B show a varied picture of how companies rated individual flexibilities with regard to their value for continued use in the post-pandemic era and their value for supporting/accelerating the development of COVID-19 therapeutics respectively. Response rates to individual flexibilities fluctuated, which may show that the actual use of certain flexibilities and guidances varied across pharmaceutical companies. Though companies were encouraged to collect input from experts across the company, an additional reason could be that the responder to this survey 
on behalf of the company was unable to engage all of the subject matter experts in preparing the response.

Some regulatory flexibilities received varied responses, which indicated that companies had varied experiences with certain flexibilities, i.e. while one company may have had a very positive experience with a particular flexibility, another company may have had a poor experience with the same flexibility. This may reflect specific characteristics of individual cases and the contexts in which they occurred. This may also reflect the "case by case" approach applied by the regulators during this period.

Nonetheless, this survey clearly shows that a number of flexibilities stood out as being considered valuable for supporting/accelerating the development of COVID-19 therapeutics and for continued use in the post-pandemic era. With regard to survey part A, this concerns the following flexibilities: ECMP (A2.1), GMP certifications (A2.3), QP/RP remote working (A2.4), direct-to-patient delivery (A3.3), remote SDV (A3.5) and GMO derogation (A3.6), which all scored particularly high overall. Moreover, flexibilities regarding variations (A1.1) and language and labelling (A1.4) were welcomed by the companies, although it was pointed out that Member States applied different approaches to implementing these flexibilities, which was considered a limiting factor in their potential value. We can summarise these flexibilities as introducing dynamic regulatory and supply arrangements, virtual and digitally supported working and collaboration across jurisdictions to support development and supply.

Due to the limited number of responses and timeframe reflected for these products, the results of part B of the survey should be considered with these limitations in mind. From the findings of survey part B, we can conclude that the flexibilities that were considered most valuable for supporting/accelerating the development of COVID-19 therapeutics, included rolling review (B1.3), the rapid agreement of a PIP and rapid compliance check (B1.4), language and labelling (B1.5), all flexibilities related to quality and supply, being ECMP (B2.1), quality variations (B2.2), GMP certifications (B2.3), QP/RP remote working (B2.4) and importation testing (B2.5). For each of these flexibilities, respondents indicated a significant value or at least some value. The flexibilities and guidances relating to rapid scientific advice (B1.2), informed consent (B3.2) and GMO derogations (B3.7) scored a high average as well, despite 'mixed' responses; while the majority of the respondents indicated significant or some value, some respondents indicated limited value. However, due to the limited number of responses, likely due to the evolving use and experience of flexibilities in support of COVID-19 therapeutics, in particular for the pharmacovigilance section, the results for survey part B should be interpretated with care.
Survey part B also showed that another contributing factor to these mixed experiences with certain flexibilities could be the timing of the use of a particular flexibility. For example, drawing these findings from the free text responses, some companies had a negative experience in the beginning of the pandemic, but later, when again making use of the same flexibility had a much better experience as some of the challenges from earlier on have been addressed. One of the examples is the experience of one company with the ETF (B1.1), which was initially considered poor, but later in the pandemic the majority of factors contributing to that experience have been addressed resulting in a much better experience by this company. This demonstrates that stakeholders reacted with agility and introduced learnings over time.

\section{Overarching Themes}

A number of overarching themes have been identified in this survey based on the learnings and experience from pharmaceutical companies with regulatory flexibilities that were considered particularly valuable:

- Making better use of digital technologies Companies generally welcomed flexibilities and guidelines that promoted the use of virtual/digital methods for executing routine tasks. Examples are flexibilities that reduce the need for on-site activities, such the flexibilities on GMP certifications (A2.3 \& B2.3), QP/RP remote working (A2.4 \& B2.4), informed consent (A3.2 \& B3.2), remote SDV (A3.5 \& B3.4) and remote PV inspections (A4.3 \& B4.3). These flexibilities offered significant value in continuing routine tasks during the pandemic, such as safeguarding clinical trial continuity as presented in other research on the implementation of regulatory flexibilities for clinical trials [19-21]. Overall these flexibilities were also considered exceptionally valuable for continued use in the post-pandemic era. Furthermore, companies expressed particular value in making more use of digital methods relating to the flexibilities regarding language and labelling (A1.4). In the context of this flexibility, respondents indicated the need to make better use of electronic product information (e.g. e-labelling) to support and speed up the continued provision of product information to patients and healthcare providers.

- Applying some flexibilities to address other healthcare challenges Respondents used the free text to express the value for some flexibilities to address other healthcare challenges, beyond the pandemic. For example, the flexibilities on language \& labelling (A1.4) and ECMP (A2.1) were considered especially valuable for continued use in the post-pandemic era for addressing drug shortages and supply constraints [22]. Furthermore, the flexibilities 
on GMO derogations (A3.6 \& B3.7) and rolling review (B1.3) were not considered extremely valuable for accelerating drug approvals in emergency situations, but were considered of value for improving the innovation climate and competitiveness of the European pharmaceutical market, ensuring quicker access to critical medicines that address unmet medical needs [23]. This echoed some of the frustration noted at the time of derogation at the failed attempts to "streamline environmental procedures among EU Member States" [24].

- Reducing the administrative burden The COVID-19 pandemic has served as a natural experiment in which some flexibilities may have proven that administrative processes need to be revised where existing regulatory requirements are regarded by manufacturers as too burdensome and/or redundant. For example, the flexibilities on quality variations (A2.2 \& B2.2), importation testing (A2.5 \& B2.5), protocol amendments (A3.1 \& B3.1) could serve as case studies to demonstrate where the current regulatory system is in a need for revision because regulatory requirements are not aligned (anymore) to the objectives they are trying to address [25]. The findings from this study show that quality flexibilities introduced by EMA have generally been welcomed by industry as helpful in enabling the development and supply of COVID-19 treatments. The learnings from this pandemic can therefore contribute to streamlining regulatory paradigms, such as the current initiatives to define innovative risk-based approaches for Chemistry, Manufacturing, and Controls (CMC) and Good Manufacturing Practices (GMPs) to accelerate the development and supply of life-saving medicines [26].

Divergent approaches across EU Member States was mentioned frequently by respondents from both survey part A and B as a limitation for many flexibilities. The lack of harmonisation between EU Member States regarding the interpretation and implementation of flexibilities and guidances at the national level was perceived as a common issue for maximizing the potential value of many flexibilities. This could also, in part, explain why some flexibilities received mixed responses with regard to their value based on positive and negative experiences. This important finding emphasises the need for regulators to consider approaches for improving the harmonisation among EU Member States prior to launching guidances and flexibilities in the future. Where this is not always possible, for example in (the beginning of) an emergency situations such as the COVID-19 pandemic, adequate measures need to be established to monitor any implementation issues at the national level in order to address these as soon as they arise. A deep-dive into case studies where a lack of harmonisation among Member States hampered the use of certain flexibilities could potentially help to get a better understanding on how to avoid this in the future. Moreover, these case studies may highlight which were success factors for situations where Member States aligned well, for example, in allowing direct to patient delivery of clinical trial therapies.

Furthermore, it is important to note in this context the activities of the International Coalition of Medicines Regulatory Authorities (ICMRA) to support the exchange of experiences and best practices relating to the use of regulatory flexibilities during the COVID pandemic. Very recently, ICMRA has started to publish these discussions for broader debate [27-29]. The opportunity remains to bring together the insights from regulators, industry as well as other stakeholders, to consider the trade-offs of benefits and risks (and costs) of regulatory flexibilities from these different perspectives. This would be an important area for further research.

\section{Limitations}

There are a number of limitations that need to be taken into account when interpreting the results of this study. These limitations are generally associated with use of online surveys for collecting data and information and are therefore also applicable to this study. For one, this survey tries to capture the experiences and learnings from pharmaceutical companies on a variety of regulatory areas with a limited set of questions. Therefore, it may not always be possible to capture the full extent of experiences that a company has on a particular topic. Moreover, the experiences with flexibilities may sometimes be dependent on a specific product/ case, which may contribute to mixed experiences with a certain flexibility. Also, the analysability and interpretation of free text fields proved challenging, albeit offering excellent insights and findings into the topics that are included in this survey. The limited number of responses to survey part B makes the analysis difficult and therefore the relevance of this survey should lie in the qualitative finding and need to be interpretated with care. It should be noted, however, that the limited number reflects in part the relatively small population of potential COVID-19 therapeutics respondents from EFPIA membership. However, despite the limited number of respondents to part B, we believe that the both surveys provide valuable insights into the regulatory flexibilities and guidances that were introduced to address the challenges from the COVID-19 pandemic. Nonetheless, it would be worthwhile to follow-up the part B analysis with a more extensive sample set at a later date.

\section{Relevance for Policy Discussions}

The results from this survey provide important insights to be used in policy debates, both at the national and the 
European level, for discussing improvements of regulatory systems based on the experiences and learnings from pharmaceutical companies and regulators from the COVID-19 pandemic. These policy discussions should help to shape future regulatory systems which is timely as Europe seeks to realise its new Pharmaceutical Strategy $^{5}$; but these policy insights should also contribute to strengthening the pandemic preparedness of the European regulatory system from the learnings of the current pandemic. This survey provides an overview of which regulatory flexibilities have been successful in achieving their goal. To the contrary this survey also provides insights into which regulatory flexibilities did not work well. Policy discussions are needed to evaluate these flexibilities and establish how these flexibilities can be improved for future use or in emergency health crisis, or to determine if regulatory resources could be better spent in other areas. Provide a more holistic overview of in-depth experiences and case studies with regulatory flexibility warrants further research. Together with the outcomes of this study and the policy discussions, this will hopefully contribute to further improving the European regulatory system to adequately address European healthcare challenges and health emergency situations such as the COVID-19 pandemic.

In conclusion, the results of this study show, for the first time, the experiences from companies with the regulatory flexibilities and guidances that were introduced to address the challenges of COVID-19 in the EU. The learning from the current pandemic presented here should contribute to policy discussions for improving the efficiency of the EU regulatory system and to strengthen the pandemic preparedness in the EU.

\section{Acknowledgements}

We want to acknowledge EFPIA members who made contributions to the industry dialogue with regulators for the regulatory flexibilities discussed here and to the research and findings herein presented; these contributors include Graham Cook (Pfizer), Veronique Devoust (Pfizer), Nick Sykes (Pfizer), Vicki Edwards (AbbVie), Esteban Herrero-Martinez (AbbVie), Nadege LeRoux (BMS), Simon Bennett (Biogen), Andrea Kurz (Roche), Rodrigo Palacios (Roche), Nicola Course (GSK), Muriel Paste (GSK), Marco Farinelli (AstraZeneca), Anne de Bock (AstraZeneca), Rose-Marie Swallow (Bayer), Aimad Torqui (MSD), Genevieve LeVisage (Novartis), Claudia Hey (Merck), Anna Czwarno (Vaccines Europe).

\section{Author Contributions}

KK and PS developed the survey instruments and research plan. The surveys were distributed to EFPIA member companies with support from EFPIA. KK collected and analysed the survey data. KK drafted the manuscript. PS, PT, VA, SM and IS reviewed the manuscript.

\footnotetext{
5 A pharmaceutical strategy for Europe I Public Health (europa.eu).
}

\section{Funding}

The work of KK and PS on this project was funded by European Federation of Pharmaceutical Industries and Associations (EFPIA). KK and PS are employed by Exon Consultancy in the Netherlands (www. exon-consultancy.nl). PT is employed by EFPIA. VA is employed by MSD. SM and IS are employed by Bayer.

\section{Declarations}

\section{Conflict of interest}

KK and PS declare that they have no conflicts of interest. PT declares that he has no conflicts of interest (besides being employed by the not for profit organisation EFPIA). VA, SM and IS are employed by pharmaceutical companies, but declare that they have no conflict of interest directly related to the article's content.

\section{Supplementary Information}

The online version contains supplementary material available at https:// doi.org/10.1007/s43441-022-00383-3.

\section{References}

1. European Medicines Agency. Treatments and vaccines for COVID-19. https://www.ema.europa.eu/en/human-regulatory/ overview/public-health-threats/coronavirus-disease-COVID-19/ treatments-vaccines-COVID-19.

2. European Commission. Communication From The Commission To The European Parliament, The Council, The European Economic And Social Committee And The Committee Of The Regions EU Strategy On COVID-19 Therapeutics. https://ec. europa.eu/info/sites/default/files/communication-strategy-covid19-therapeutics_en.pdf.

3. LaVange L, Adam SJ, Currier JS, et al. Accelerating COVID-19 Therapeutic Interventions and Vaccines (ACTIV): designing master protocols for evaluation of candidate COVID-19 therapeutics. Ann Intern Med. 2021;174:1293-300.

4. Bolislis WR, de Lucia ML, Dolz F, et al. Regulatory agilities in the time of COVID-19: overview, trends, and opportunities. Clin Ther. 2021;43:124-39.

5. Chong SSF, Kim M, Limoli M, et al. Measuring progress of regulatory convergence and cooperation among Asia-Pacific Economic Cooperation (APEC) member economies in the context of the COVID-19 pandemic. Ther Innov Regul Sci. 2021;55:786-98.

6. Forman R, Atun R, McKee M, et al. 12 Lessons learned from the management of the coronavirus pandemic. Health Policy. 2020;124:577-80.

7. European Commission. Notice to stakeholders questions and answers on regulatory expectations for medicinal products for human use during The COVID-19 pandemic. https://ec.europa.eu/ health/sites/default/files/human-use/docs/guidance_regulatory_ covid19_en.pdf.

8. The Co-ordination Group for Mutual Recognition and Decentralised Procedures - human (CMDh). Practical Guidance Of The CMDh For Facilitating The Handling Of Processes During The COVID-19 Crisis. https://www.hma.eu/fileadmin/dateien/Human Medicines/CMD_h_/COVID-19/CMDh_418_220_Rev1_05_ 2020_clean_-_PG_on_COVID-19_crisis.pdf. 
9. European Commission. Guidance on the management of clinical trials during the COVID-19 (Coronavirus) pandemic. https:// ec.europa.eu/health/sites/default/files/files/eudralex/vol-10/guida nceclinicaltrials_covid19_en.pdf.

10. European Commission. Regulation (EU) 2020/1043 Of The European Parliament And Of The Council Of 15 July 2020 On The Conduct Of Clinical Trials With And Supply Of Medicinal Products For Human Use Containing Or Consisting Of Genetically Modified Organisms Intended To Treat Or Prevent Coronavirus Disease (COVID-19). https://eur-lex.europa.eu/legal-content/EN/ $\mathrm{TXT} / \mathrm{PDF} /$ ?uri=CELEX:32020R1043\&from $=\mathrm{EN}$.

11. European Medicines Agency. Mandate, objectives and rules of procedure of the COVID-19 EMA pandemic Task Force (COVIDETF). https://www.ema.europa.eu/en/documents/other/mandateobjectives-rules-procedure-covid-19-ema-pandemic-task-forcecovid-etf_en.pdf.

12. European Medicines Agency. EMA initiatives for acceleration of development support and evaluation procedures for COVID-19 treatments and vaccines. https://www.ema.europa.eu/en/docum ents/other/ema-initiatives-acceleration-development-supportevaluation-procedures-covid-19-treatments-vaccines_en.pdf.

13. European Medicines Agency. Labelling flexibilities for COVID19 therapeutics. https://www.ema.europa.eu/en/documents/other/ labelling-flexibilities-covid-19-therapeutics_en.pdf.

14. Food and Drug Administration/European Medicines Agency. FDA/EMA Common Commentary on Submitting an initial Pediatric Study Plan (iPSP) and Paediatric Investigation Plan (PIP) for the Prevention and Treatment of COVID-19.

15. Chyung SYY, Roberts K, Swanson I, et al. Evidence-based survey design: the use of a midpoint on the likert scale. Perform Improv. 2017;56:15-23.

16. European Medicines Agency. COVID-19 treatments. https://www. ema.europa.eu/en/human-regulatory/overview/public-health-threa ts/coronavirus-disease-covid-19/treatments-vaccines/covid-19treatments.

17. Garbe JHO, Jacobs MG, Rönninger SK. Import testing: an outdated practice? Opportunities for improved access to safe and efficient medicines. Ther Innov Regul Sci. 2017;51:620-4.

18. International Federation of Pharmaceutical Manufacturers \& Associations (IFPMA). IFPMA policy position-best practices for in-country testing and sample management. https://www. ifpma.org/wp-content/uploads/2020/10/Sample-Mgmt_Joint-Posit ion_final.pdf.

19. Jong AJ, Santa-Ana-Tellez Y, Thiel GJMW, Zuidgeest MGP, Siiskonen SJ, Mistry D, et al. COVID-19 and the emerging regulatory guidance for ongoing clinical trials in the European Union. Clin Pharmacol Ther. 2021;109:1517-27.
20. Upadhaya S, Yu JX, Oliva C, Hooton M, Hodge J, Hubbard-Lucey VM. Impact of COVID-19 on oncology clinical trials. Nat Rev Drug Discov. 2020;19:376-7.

21. Xue JZ, Smietana K, Poda P, Webster K, Yang G, Agrawal G. Clinical trial recovery from COVID-19 disruption. Nat Rev Drug Discov. 2020;19:662-3.

22. European Federation of Pharmaceutical Industries and Associations. Reflection paper on regulatory mitigation measures for shortages of medicinal products. https://www.efpia.eu/media/ 554740/reflection-paper-on-regulatory-mitigation-measures-forshortages-of-medicinal-products.pdf.

23. Alliance for Regenerative Medicine (ARM), European Federation of Pharmaceutical Industries and Associations (EFPIA), European Association for Bioindustries (EuropaBio), Beattie S, Hubert A, Morrell J, et al. Call for more effective regulation of clinical trials with advanced therapy medicinal products consisting of or containing genetically modified organisms in the European Union. Hum Gene Ther. 2021; hum.2021.058.

24. Iglesias-Lopez C. Temporary derogation from European environmental legislation for clinical trials of genetically modified organisms for coronavirus disease 2019. Cytotherapy. 2021;23:10-1.

25. Klein K, Stolk P, De Bruin ML, et al. Regulatory density as a means to refine current regulatory approaches for increasingly complex medicines. Drug Discov Today. 2021;26:2221-5.

26. European Federation of Pharmaceutical Industries and Associations. EFPIA White Paper on CMC development, manufacture and supply of pandemic COVID-19 therapies and vaccines. https:// www.efpia.eu/media/554681/cmc-development-manufacture-andsupply-of-COVID-19-therapies-and-vaccines.pdf.

27. International Coalition of Medicines Regulatory Authorities (ICMRA). Report on the review of regulatory flexibilities/agilities as implemented by National Regulatory Authorities during Covid-19 pandemic_December 2020. https://icmra.info/drupal/ sites/default/files/2021-12/Regulatory_Flexibilities_during_ COVID-19_Report.pdf.

28. International Coalition of Medicines Regulatory Authorities (ICMRA). Deep dive report on the review of provisions and procedures for emergency authorization of medical products for COVID-19 among ICMRA members-July 2021. https://icmra. info/drupal/sites/default/files/2021-12/eua_deep_dive_report.pdf.

29. International Coalition of Medicines Regulatory Authorities (ICMRA). Reflections on the regulatory experience of remote approaches to GCP and GMP regulatory oversight during the COVID-19 Pandemic. https://icmra.info/drupal/sites/default/files/ 2021-12/remote_inspections_reflection_paper.pdf. 